\title{
Factores condicionantes de la transferencia de la formación permanente del profesorado no universitario.
}

\section{Conditioning factors for the transfer of permanent training for non- university teachers.}

\author{
Marcial Pamies Berenguer \\ Universidad de Murcia. Murcia, España \\ marcial.pamies@um.es \\ María Ángeles Gomariz Vicente \\ Universidad de Murcia. Murcia, España \\ magovi@um.es \\ Antonia Cascales Martínez \\ Universidad de Murcia. Murcia, España \\ antonia.cascales@um.es
}

\begin{abstract}
Resumen
Los planes de desarrollo profesional de los docentes constituyen el mecanismo principal de actuación para la mejora de los servicios ofrecidos por las organizaciones educativas y del desempeño de las funciones del profesorado. El estudio de la transferencia de los aprendizajes desarrollados en las actividades de formación permanente al puesto de trabajo configura un problema de gran interés al tratarse de un elemento necesario para poder analizar el rendimiento de las actividades formativas. La presente investigación explora la estructura factorial condicionante de la transferencia de la formación permanente del profesorado no universitario, analiza las analogías y diferencias con otros modelos y propone un instrumento de medición adaptado a la enseñanza no universitaria. La metodología utilizada obedece a un diseño no experimental de tipo cuantitativo basado en un diseño transversal tipo encuesta. La muestra está formada por 4769 docentes no universitarios de la Región de Murcia, obtenidos mediante un muestreo no probabilístico por conveniencia. El análisis ha seguido un enfoque cuantitativocorrelacional empleando técnicas estadísticas de análisis factorial y el estudio de la consistencia interna. La investigación ha permitido proponer un modelo factorial y un instrumento de medida, validado en contenido y constructo para los factores de transferencia en la enseñanza no universitaria.
\end{abstract}

Palabras clave: Entrenamiento Profesional, Transferencia de Formación, Aprendizaje Permanente, Análisis Factorial, Profesorado.

\begin{abstract}
Teachers' professional development plans constitute the main mechanism of action to improve the services offered by educational organizations and the performance of the functions of teachers. The study of the transfer of learning developed in permanent training activities to the job constitutes a problem of great interest as it is a necessary element to be able to analyze the performance of training activities. This research explores the factorial structure that determines the transfer of permanent training for non-university teachers, analyzes the analogies and differences with other models and proposes a measurement instrument adapted to non-university teaching. The methodology used follows a non-experimental, quantitative design based on a cross-sectional survey-type design. The sample is made up of 4,769 non-university teachers from the Region of Murcia, obtained through a non-probabilistic convenience sampling. The analysis followed a quantitative-correlational approach using statistical factor analysis techniques and the study of internal consistency. The research has made it possible to propose a
\end{abstract}

RED. Revista de Educación a Distancia. Núm. 69, Vol. 22. Artíc. 4, 30-Ene-2022

DOI: http://dx.doi.org/10.6018/red.486801 
factorial model and a measurement instrument, validated in content and construct, for transfer factors in non-university education.

Key words: Professional Training, Transfer of Training, Lifelong Learning, Factor Analysis, Teachers.

\section{Introducción}

Desde un punto de vista general, el desarrollo profesional de los empleados de una organización, entendido como la formación de carácter permanente que realizan los empleados a lo largo de su vida profesional con la finalidad de incorporar, actualizar o desarrollar conocimientos, destrezas y actitudes que les permitan mejorar su labor en el puesto de trabajo y las operaciones de la propia organización (Comisión Europea/EACEA/Eurydice, 2018), forma parte de las prácticas habituales, que se encuentran a disposición de los departamentos encargados de la gestión de los Recursos Humanos de las organizaciones, para introducir mejoras en el rendimiento y la calidad de los servicios o productos ofertados (Anwar y Abdullah, 2021).

La formación permanente del profesorado es un derecho del profesional de la educación y un deber de la administración, según la normativa española y, por ello, se concibe como una herramienta necesaria para el desarrollo profesional, a la vez que un factor esencial, en la medida que se tiene que habilitar para enfrentar nuevas fórmulas de trabajo y, en consecuencia, nuevas tareas que mejoren la calidad de la enseñanza (Feixas et al., 2013a). Los programas de formación docente se constituyen en posibilidades pedagógicas sólidas y relevantes para la conformación de equipos profesionales independientes, autónomos, competentes y actualizados; con el fin de impactar con eficacia en el aprendizaje del alumnado y desarrollar con éxito la práctica docente (Buckworth, 2017; Canto, 2016; García et al., 2020; Hicks, 1999; SalazarGómez y Tobon, 2018). La finalidad de estos programas de formación es que el aprendizaje obtenido pueda aplicarse en la función docente, es decir, la transferencia al puesto de trabajo; ello supone una doble función, por un lado, la adquisición de conocimientos, habilidades y actitudes y, por otro, la aplicación continuada en el tiempo (Baldwin y Ford, 1988; Cejas-León y Navío-Gámez, 2020; Nafukho et al., 2017).

La transferencia de conocimiento es una máxima en la sociedad contemporánea (Díaz et al., 2021), en el caso de la transferencia de la formación dicha máxima se fundamenta sobre la convicción de que la formación y el desarrollo mejoran la competitividad organizacional (Ford et al., 2018). La transferencia de formación es un concepto complejo y dinámico. Se puede definir como la generalización al contexto laboral y el mantenimiento a lo largo del tiempo, de la aplicación efectiva de los conocimientos y habilidades adquiridas en un contexto de aprendizaje (Baldwin y Ford, 1988; Baldwin et al., 2009; Freitas et al., 2017; Govaerts et al., 2017; Newstrom, 1986).

Ciertamente, se han realizado muchas investigaciones, no tanto en promover y evaluar la transferencia de la formación sino en la medición de los factores condicionantes (Baldwin et al., 2017; Bawono y Purnomo, 2016; Feixas et al., 2013a; Gafas González et al., 2018; García et al., 2018; Vélez Álvarez et al., 2018). En esa necesidad de identificar los factores que influyen en la transferencia de la formación, diferentes autores determinaron que dichos factores se pueden clasificar en tres grupos: factores personales, factores de la formación y factores del contexto (Baldwin y Ford 1988; Feixas et al., 2015; Parra Robledo y Ruiz Bueno, 2020).

Factores condicionantes de la transferencia de la formación permanente del profesorado no universitario. Pamies-Berenguer M., Gomariz-Vicente M. A. y Cascales-Martínez A. 
Con respecto a los factores personales, se refieren a las percepciones de los docentes participantes centrados en su desarrollo personal (Cejas y Navío, 2017; Parra Robledo y Ruiz Bueno, 2020; Salazar-Gómez y Tobon, 2018), concretamente a su capacidad para transferir, motivar y empatiza (Feixas et al., 2015).

Respecto a los factores de la formación, según Feixas et al. (2015), se refieren al diseño de la formación y al aprendizaje realizado. Parra Robledo y Ruiz Bueno (2020) concretan el diseño de la formación a la promoción de la reflexión pedagógica en los contenidos tratados, la consecución de objetivos de la formación, las estrategias metodológicas y un clima favorable para el aprendizaje.

Finalmente, los factores del contexto se relacionan con el apoyo del responsable, predisposición al cambio, los recursos del entorno, retroalimentación por parte del docente participante, reconocimiento institucional y cultura docente de equipo de trabajo (Feixas et al., 2015). Parra Robledo y Ruiz Bueno (2020), asocian estos factores a la aplicación de la formación y el desarrollo competencial en el puesto de trabajo, destacando la contribución de la formación en la mejora del clima laboral y la facilitación de la transferencia al entorno de trabajo.

\subsection{Antecedentes y preguntas de investigación}

A la vista del marco teórico descrito anteriormente, se hace patente que el estudio sobre los factores que influyen en la transferencia de la formación permanente al puesto de trabajo ha sido una cuestión recurrente en la literatura científica de la temática. Estas investigaciones no sólo se han diferenciado en el enfoque empleado para el diseño de la investigación, sino que han jugado un papel muy importante las particularidades del puesto de trabajo en el que se enmarcaba el estudio, o las asociadas al diseño de la propia formación. Aunque la tendencia actual centra, entre otros, el estudio de la transferencia en investigar las características e intereses personales de los consumidores de la formación permanente o la manera en la que éstos diseñan sus propios itinerarios formativos (Baldwin et al., 2017; Poell, 2017), se considera que la identificación de estructuras factoriales asociadas al proceso de estudio en contextos profesionales concretos aporta, por un lado, una mejor comprensión de la situación de estudio en dicho contexto particular $\mathrm{y}$, por otro, contribuye al proceso de generalización de estructuras ya estudiadas en contextos afines.

En el contexto particular de la formación continua del docente, Feixas et al. (2013a) presentan un instrumento de medida para los factores de influencia en la transferencia de la formación del profesorado universitario, proponiéndose una estructura factorial para dicho constructo formada por 8 factores. Con el objetivo de adaptar dicho instrumento, explorar diferentes modelos factoriales derivados del nuevo instrumento y analizar la robustez del modelo factorial primigenio al trasladarlo, de forma adaptada, al escenario de la formación permanente del profesorado no universitario, en PamiesBerenguer et al. (2020a, 2020b) se desarrolló el proceso de validación de contenido de un instrumento de medida adaptado a la enseñanza no universitaria. Al mismo tiempo que se realizó la validación de contenido del cuestionario, por un grupo de 15 expertos ( 3 mujeres y 12 hombres) que desarrollaban su actividad profesional o investigadora en universidades españolas (13) o en Centros de Formación del profesorado (2), con una gran experiencia profesional $(M=20.8$ años, $D T=8.8$ años) y cuya temáticas de investigación o profesional se alineaban con la de la investigación propuesta: Formación

Factores condicionantes de la transferencia de la formación permanente del profesorado no universitario. Pamies-Berenguer M., Gomariz-Vicente M. A. y Cascales-Martínez A. 
del profesorado universitario (7); Formación del profesorado no universitario (3); Transferencia de la formación (5); Organización Escolar (1); Didáctica (4). Estos expertos indicaron la adscripción de cada una de las cuestiones propuestas a uno de los factores propuestos en el modelo de Feixas et al. (2013b). Así, se fijaron para este trabajo los objetivos de explorar la estructura factorial asociada al marco de la educación no universitaria, investigar su robustez y proponer un instrumento de medición adaptado a dicho marco.

Teniendo presentes los objetivos iniciales de la investigación y los antecedentes obtenidos durante el proceso descritos anteriormente, se concretaron las siguientes preguntas de investigación:

P1. ¿Es posible trasladar el modelo factorial desde el marco de la educación universitaria al de la no universitaria?

P2. ¿Es posible derivar un instrumento para la medición de los factores que sea compatible con el modelo trasladado a la enseñanza no universitaria o, por el contrario, se adapta mejor a una nueva estructura factorial? En caso de que aparezca una nueva estructura ¿cómo se describe en términos de factores de influencia sobre la transferencia?

\section{Materiales y métodos}

\subsection{Diseño y participantes}

La investigación obedece a un diseño no experimental, exploratorio, de carácter descriptivo, adoptando un diseño transversal tipo encuesta para describir, analizar e interpretar un conjunto de datos y variables sobre una realidad concreta de la manera más objetiva y comprobable posible (Cubo et al., 2018). Se ha contado con la participación de 4769 docentes de la Región de Murcia, un $77.4 \%$ de los cuales son mujeres y un $22.6 \%$ hombres. Atendiendo a los puestos docentes de los participantes, un 9.4\% indica haber recibido formación específica para equipos directivos de centros docentes, el 13.6\% accedieron a la formación como tutoras/es de grupos de alumnos y el resto como profesorado de diferentes niveles y asignaturas. La Tabla 1 muestra los datos asociados a la distribución de la muestra según el rango de edad, tipo de centro en el que se imparte docencia y la etapa educativa (en esta última variable se muestran sólo aquellos datos con mayor frecuencia). Los docentes participantes fueron seleccionados a través de un muestreo no probabilístico por conveniencia entre aquellos docentes que realizaron alguna actividad formativa programada por el Centro de Profesores y Recursos de la Región de Murcia (CPR Región de Murcia) durante los cursos 20182019 y $2019-2020$.

\subsection{Instrumento}

El instrumento se configuró usando el banco de preguntas cuya validación de contenido se realizó en la referencia Pamies-Berenguer et al. (2020a). Las preguntas fueron incorporadas a un cuestionario online, cuyo esquema se puede consultar en la referencia Pamies-Berenguer et al. (2020b), que se enlazó mediante los cuestionarios propios del

Factores condicionantes de la transferencia de la formación permanente del profesorado no universitario. Pamies-Berenguer M., Gomariz-Vicente M. A. y Cascales-Martínez A. 
CPR Región de Murcia que se utilizan para la evaluación interna de las actividades formativas y los programas de formación incluidos en los Planes Regionales de Formación Permanente del profesorado.

El cuestionario se dividió en tres bloques. En el primer bloque de preguntas se solicitaban datos de carácter sociodemográfico (Edad, Sexo, Años de Experiencia, Puesto docente, Nivel que imparte, Situación laboral, ...) y de contexto, cuyas preguntas (programas de formación asociados a las actividades realizadas, objetivos enfocados dentro de los diferentes planes de formación, ...) se encontraban relacionadas con los Planes de Formación y objetivos recogidos en el Plan Trienal de Formación Permanente del Profesorado 2018-2020 aprobado por la Orden 6771/2018 de 30 de octubre de 2018 de la Consejería de Educación, Juventud y Deportes. A continuación, se presentaron las 56 preguntas propias del cuestionario de factores influyentes de transferencia de la formación permanente del profesorado no universitario (ver Apéndice), terminando con un bloque de recogida de datos dedicado a la estimación de la aplicación de los aprendizajes al puesto de trabajo: estimación de la frecuencia de oportunidades para la aplicación de conocimientos, actitudes o destrezas y la estimación del porcentaje en el que se produjo un aplicación real al detectarse las condiciones para la aplicación. Ambas cuestiones fueron medidas utilizando la escala de rangos de porcentaje: $0 \%$ $20 \% ; 20 \%-40 \% ; 40 \%$ - 60\%; 60\% - 80\% y 80\% - $100 \%$. Por último, se solicitó una estimación subjetiva del grado de transferencia en una escala Likert (1-10).

Tabla 1.

Variables sociodemográficas de los docentes participantes en el estudio.

\begin{tabular}{|c|c|c|c|}
\hline Variable & Clases & Frecuencia & Porcentaje \\
\hline \multirow{4}{*}{ Edad } & Menos de 30 años & 339 & $7.1 \%$ \\
\hline & $30-40$ & 1289 & $27.0 \%$ \\
\hline & $40-50$ & 1748 & $36.7 \%$ \\
\hline & Más de 50 años & 1393 & $29.2 \%$ \\
\hline \multirow{3}{*}{$\begin{array}{l}\text { Tipo de } \\
\text { Centro }\end{array}$} & Público & 4078 & $85.5 \%$ \\
\hline & Concertado & 651 & $13.6 \%$ \\
\hline & Privado & 7 & $0.1 \%$ \\
\hline \multirow{7}{*}{$\begin{array}{c}\text { Etapa } \\
\text { Educativa }\end{array}$} & Infantil & 652 & $13.7 \%$ \\
\hline & Primaria & 1326 & $27.8 \%$ \\
\hline & Infantil y Primaria & 700 & $14.7 \%$ \\
\hline & ESO & 470 & $9.9 \%$ \\
\hline & ESO y Bachillerato & 856 & $17.9 \%$ \\
\hline & Formación Profesional & 352 & $7.4 \%$ \\
\hline & Educación Especial & 147 & $3.1 \%$ \\
\hline
\end{tabular}

Fuente: elaboración propia

Factores condicionantes de la transferencia de la formación permanente del profesorado no universitario. Pamies-Berenguer M., Gomariz-Vicente M. A. y Cascales-Martínez A. 
El instrumento contenía un texto de bienvenida, que explicaba los objetivos de la investigación, las particularidades del almacenamiento y tratamiento de los datos, la política de confidencialidad y los compromisos adquiridos por los participantes y los investigadores. Asimismo, se dispuso de un texto de despedida que agradecía la disposición y el interés en participar en el proceso de recogida de datos.

\subsection{Procedimiento}

Previo a realizar el estudio, se informó y se solicitó colaboración al área de responsables de la formación permanente del profesorado de la Consejería de Educación de la Comunidad Autónoma de la Región de Murcia (España), con quien se firmó un acuerdo de colaboración para realizar el estudio. La información del estudio se remitió al Comité de Ética de la Universidad de Murcia, dando aprobación a su desarrollo. A continuación, se mantuvieron reuniones con los responsables del CPR Región de Murcia en las que se acordó remitir el enlace al cuestionario directamente a todos los participantes que hubieran realizado alguna actividad formativa durante el curso 20182019 y distribuirlo mediante los cuestionarios de evaluación final que los participantes realizan al concluir las actividades formativas a aquellos que realizaron la formación durante el curso 2019-2020.

\subsection{Análisis de datos}

Los ítems de la escala empleada durante la fase de recogida de datos estaban configurados como escala tipo Likert (1-5). Así, los métodos de análisis factorial, tanto exploratorio como confirmatorio, se realizaron tomando como base el uso de la matriz de correlación policórica y las técnicas de estimación e inferencia asociadas al uso de variables ordinales (Izquierdo et al., 2014).

El procedimiento consistió en la aplicación de un análisis factorial exploratorio (AFE), con el fin de obtener modelos factoriales plausibles para la estructura de variables latentes asociadas, seguido de la realización de un análisis factorial confirmatorio (AFC), con el objetivo de disponer de métricas estadísticas sobre la bondad del ajuste de cada modelo. Durante la fase de análisis factorial confirmatorio, se añadió el modelo teórico adaptado durante la fase de validación de expertos del propuesto por Feixas et al. (2013a). Por último, se seleccionó el modelo de mejor ajuste y se estudió la consistencia interna de los ítems que formaron cada uno de los factores. La muestra inicial de 4769 datos se dividió en dos conjuntos, MAFE de $\mathrm{n}_{1}=2454$ individuos para realizar el AFE y muestra MAFC de $\mathrm{n}_{2}=2315$ para la realización del AFC y el estudio de la consistencia interna mediante el alfa de Cronbach. Las asignaciones a los grupos se realizaron utilizando la función sample del paquete base de R Commander.

Previo al análisis exploratorio de los datos se invirtieron los valores, de todos los datos muestrales, asociados a las cuestiones redactadas en sentido negativo (ítem 21, 22, 23, 37, 40, 42, 46 y 47) del cuestionario de transferencia de la formación permanente del profesorado no universitario (Pamies-Berenguer et al., 2020a, 2020b) que se utilizó como instrumento de recogida de datos.

Para la realización de los diferentes análisis se utilizó el software estadístico R en su versión 4.0.2 (R Core Team, 2020), ejecutado en el sistema operativo macOS Sierra

Factores condicionantes de la transferencia de la formación permanente del profesorado no universitario. Pamies-Berenguer M., Gomariz-Vicente M. A. y Cascales-Martínez A. 
versión 10.12.6. El análisis factorial exploratorio se realizó utilizando los paquetes "parameters" (Lüdecke et al., 2020), "psych" (Revelle, 2020), "EFA.MRFA" (NavarroGonzalez y Lorenzo-Seva, 2020) y "GPArotation" (Bernaards y Jennrich, 2005). Durante la fase de análisis factorial confirmatorio se emplearon los paquetes "lavaan" (Rosseel, 2012). Por último, se utilizó el paquete "psych" para el estudio de la consistencia interna de las agrupaciones de ítems en el modelo factorial resultante con el estadístico alfa de Cronbach.

Con el fin de estudiar un número amplio de modelos y no descartar posibilidades en el número de factores, la fase exploratoria se desarrolló de forma iterativa identificando el número de factores propuestos por diferentes métodos (Análisis Paralelo, MAP de Velicer, BIC, TLI, RMSA, Criterio de Kaiser, Métodos de Bentler, Bartlett, Anderson y Lawley, ...), se decidieron los números a explorar aplicando un sistema de puntaje de los números obtenidos por los diferentes métodos y se combinaron los métodos de estimación factorial de máxima verosimilitud (ML), mínimos cuadrados ordinario (OLS), mínimos cuadrados no ponderado (ULS) y de rango mínimo (MINRANK) con las estrategias de rotación varimax, oblimin y promin. La Figura 1 muestra, de forma esquemática, los pasos realizados en cada iteración del procedimiento.

En la fase AFC se sometieron a estudio los 8 modelos encontrados durante la fase exploratoria y el modelo teórico adaptado del contexto universitario. La bondad del ajuste se evaluó utilizando diferentes índices: el error cuadrático medio de aproximación (RMSEA), el índice de ajuste comparativo (CFI), el índice de Tucker-Lewis (TLI), el residuo estándar cuadrático medio (SRMR) y los criterios para la toma de decisiones siguientes (Hu y Bentler,1999):

- RMSEA<.05 (o SRMR<.05) en combinación con CFI>.95 y TLI>.95, se corresponden con un ajuste del modelo muy ajustado.

- . $.05<$ RMSEA<.08 (ó .05<SRMR<.08) en combinación con .95<CFI y .95<TLI, se corresponden con ajustes del modelo bueno.

- $.05<$ RMSEA<.08 (ó .05<SRMR<.08) en combinación con $.90<$ CFI y .90<TLI, se corresponden con ajustes del modelo adecuado.

Debido al uso de datos ordinales y la matriz de correlaciones policórica, el ajuste de los modelos en la fase confirmatoria se realizó usando el método de estimación por mínimos cuadrados ponderados con media y varianza ajustada (WLSMV).

\section{Resultados}

\subsection{Análisis factorial exploratorio}

El análisis factorial exploratorio se realizó utilizando las respuestas a los 56 ítems recogidos en el bloque "Cuestionario sobre la transferencia de la formación permanente del profesorado no universitario" del cuestionario Pamies-Berenguer et al. (2020b) ofrecidas por los 2454 individuos asignados a la muestra MAFE. Los estadísticos asociados al análisis multivariado de la asimetría y curtosis (Mardia, 1970) (Mardia_Skewness $=120933.30 \mathrm{p}$-value $<.000 ;$ Mardia_Kurtosis $=422.03$ p-value $<$ .000) apuntan hacia la no normalidad de la distribución subyacente a las puntuaciones,

Factores condicionantes de la transferencia de la formación permanente del profesorado no universitario. Pamies-Berenguer M., Gomariz-Vicente M. A. y Cascales-Martínez A. 
apoyando la decisión de utilizar la matriz policórica como base para el proceso de factorización. Las medidas de adecuación de la matriz de correlación para el análisis factorial ofrecidas por el test de esfericidad de Bartlett $(\mathrm{B}=153003.3 ; \mathrm{gl}=1540 \mathrm{p}$-value $<.000)$ y el test de adecuación de Kaiser-Meyer-Olkin $(\mathrm{KMO}=.9709439>$ > 9$)$ apuntan hacia una situación de partida bien adaptada para la aplicación de dicho análisis.

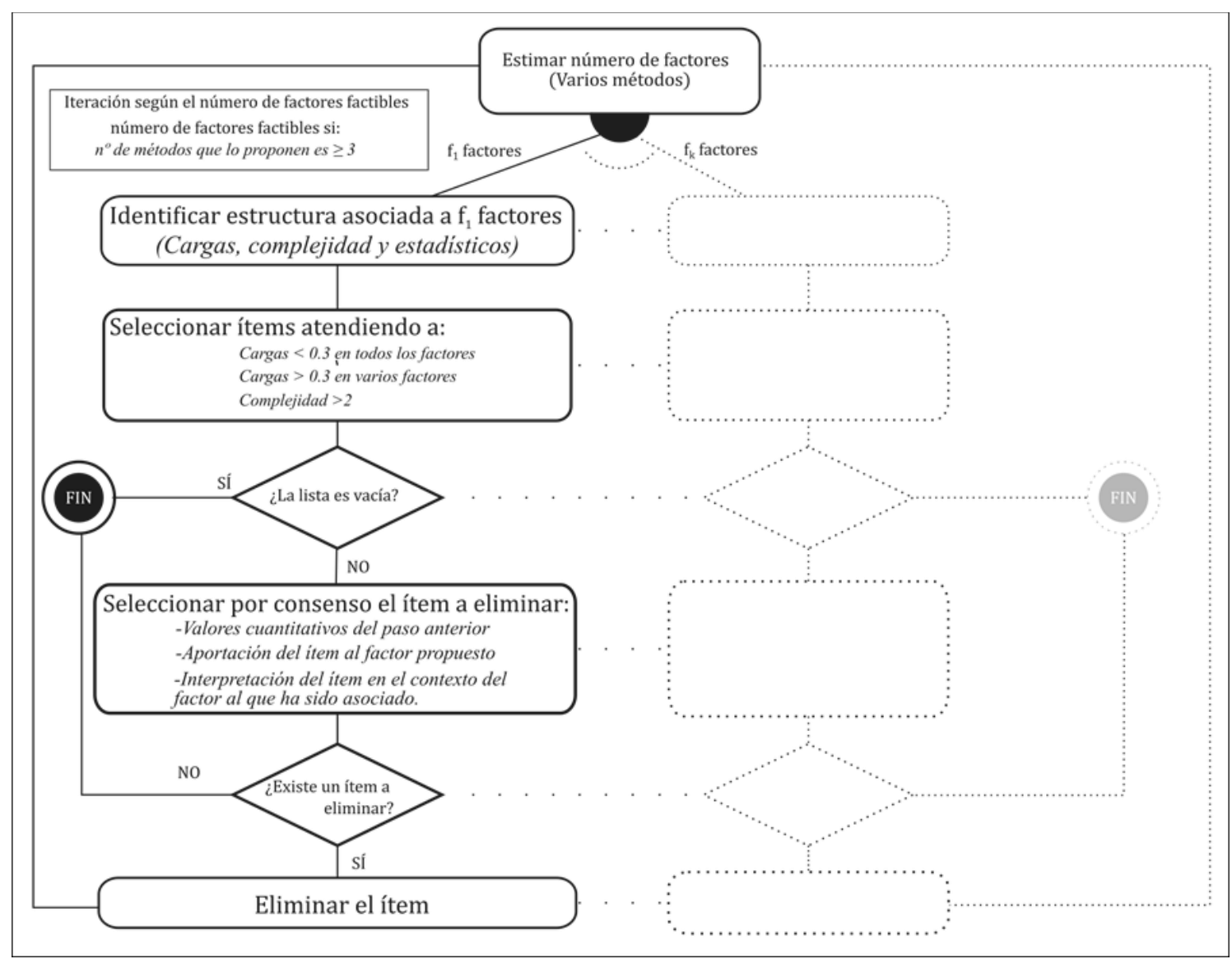

Figura 1. Diagrama esquemático del proceso iterativo utilizado en el análisis factorial exploratorio. Fuente: elaboración propia.

El valor del determinante de la matriz de correlaciones resultó un valor cercano a cero, lo que podría estar ligado a un efecto de multicolinealidad. Sin embargo, el análisis de los factores de inflación de la varianza (VIF) de cada una de los ítems, calculados introduciendo una variable sintética a modo de variable dependiente, resultaron por debajo $10(\max (\mathrm{VIFi})<7.1)$, valor límite considerado por algunos autores (Kleinbaum et al., 1988; Mason et al., 1989) como umbral mínimo para considerar el efecto de la multicolinealidad como adverso.

La Tabla 2 muestra los datos estadísticos obtenidos por las diferentes combinaciones de métodos de estimación y estrategias de rotación tras aplicar el procedimiento iterativo. El número de ítems eliminados siguiendo el criterio de salida (Carga <.3; Complejidad $>2$; baja expresividad) varió en función a la combinación (Método de estimaciónEstrategia de rotación) adoptada.

Factores condicionantes de la transferencia de la formación permanente del profesorado no universitario. Pamies-Berenguer M., Gomariz-Vicente M. A. y Cascales-Martínez A. 
Tabla 2.

Resultados del análisis factorial exploratorio. Estadísticos distribuidos según la combinación Método de Estimación-Rotación

\begin{tabular}{|c|c|c|}
\hline M. Estimación & Rotación & Estadísticos \\
\hline \multirow{16}{*}{ OLS } & \multirow{8}{*}{ VARIMAX } & N. Factores $=4$ \\
\hline & & Ítems eliminados $=15$ \\
\hline & & Iteraciones $=16$ \\
\hline & & $\chi^{2}=114257.5$ \\
\hline & & $\mathrm{TLI}=.807$ \\
\hline & & $\mathrm{RMSEA}=.104$ \\
\hline & & $\mathrm{SRMR}=.04$ \\
\hline & & $\mathrm{BIC}=13114.84$ \\
\hline & \multirow{8}{*}{ OBLIMIN } & N. Factores $=7$ \\
\hline & & Ítems eliminados $=11$ \\
\hline & & Iteraciones $=12$ \\
\hline & & $\chi^{2}=125518.5$ \\
\hline & & $\mathrm{TLI}=.863$ \\
\hline & & RMSEA $=.084$ \\
\hline & & $\mathrm{SRMR}=.02$ \\
\hline & & $\mathrm{BIC}=7192.91$ \\
\hline \multirow{13}{*}{ ULS } & \multirow{13}{*}{ OBLIMIN } & N. Factores $=6$ \\
\hline & & Ítems eliminados $=10$ \\
\hline & & Iteraciones $=11$ \\
\hline & & $\chi^{2}=127040.3$ \\
\hline & & $\mathrm{TLI}=.847$ \\
\hline & & RMSEA $=.087$ \\
\hline & & SRMR $=.02$ \\
\hline & & $\mathrm{BIC}=9119.71$ \\
\hline & & N. Factores $=7$ \\
\hline & & Ítems eliminados $=11$ \\
\hline & & Iteraciones $=12$ \\
\hline & & $\chi^{2}=124809.8$ \\
\hline & & $\mathrm{TLI}=.866$ \\
\hline
\end{tabular}

Factores condicionantes de la transferencia de la formación permanente del profesorado no universitario. Pamies-Berenguer M., Gomariz-Vicente M. A. y Cascales-Martínez A. 


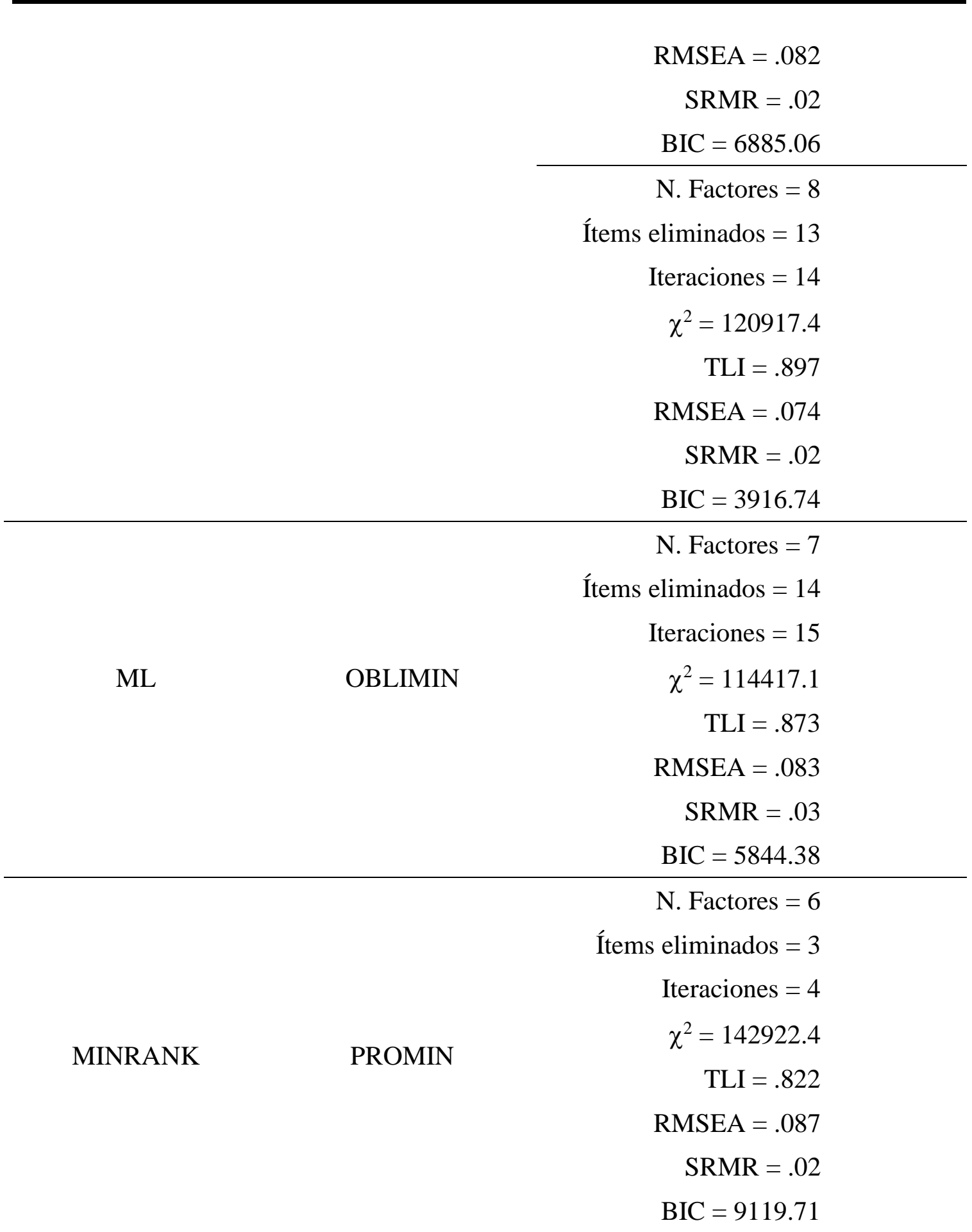

Nota. Fact. $=$ número de factores obtenidos; Elim. $=$ número de ítems eliminados en el proceso iterativo; I. max = número de iteraciones máxima realizadas en el proceso descrito en la Figura $1 ; \chi^{2}=$ estadístico Chi-cuadrado;

TLI = Tucker-Lewis Index; RMSEA = Error cuadrático medio de aproximación; SRMR $=$ Residuo estandarizado cuadrático medio $;$ BIC $=$ Bayesian Information Criterion.

Fuente: elaboración propia.

Factores condicionantes de la transferencia de la formación permanente del profesorado no universitario. Pamies-Berenguer M., Gomariz-Vicente M. A. y Cascales-Martínez A. 
A la vista de los resultados, se decidió someter todos los modelos obtenidos al estudio de la bondad de ajuste en la fase de análisis factorial confirmatorio.

\subsection{Análisis factorial confirmatorio}

Durante el análisis factorial confirmatorio, se utilizó la muestra de 2215 individuos MAFC y se sometió a estudio el modelo de un factor, los siete modelos descritos en la Tabla 1 y el modelo teórico adaptado del propuesto para el escenario de la educación no universitaria (ver Figura 2). La Tabla 3 muestra los valores que se obtuvieron para los índices de bondad de ajuste de cada modelo, utilizando el método de estimación de por mínimos cuadrados ponderados con media y varianza ajustada. Atendiendo a los criterios de selección se observó que el modelo ULS-OBLIMIN de 8 factores presentaba una combinación de índices en los que CFI $>.95$ y TLI $>95$ y SRMR $<.05$, cumpliendo el criterio propuesto para considerar el modelo como de muy ajustado. Por otra parte, los modelos de 4, 6 y 7 factores presentaron una combinación de índices que los catalogaron dentro de los modelos adecuados CFI $>.95$ y TLI $>.95$ con $.05 \leq$ SRMR $<.08$ (ó $.05 \leq$ RMSEA <.08). Los modelos de un factor y el modelo de 8 factores adaptado del marco no universitario (asumiendo la adscripción de las cuestiones a los factores propuestos) se correspondieron con los modelos menos adecuados en términos de bondad de ajuste.

\section{Tabla 3.}

Resultados del análisis factorial confirmatorio. Estadísticos de bondad de ajuste de los modelos factoriales propuestos en la fase exploratoria y el modelo teórico propuesto por los expertos.

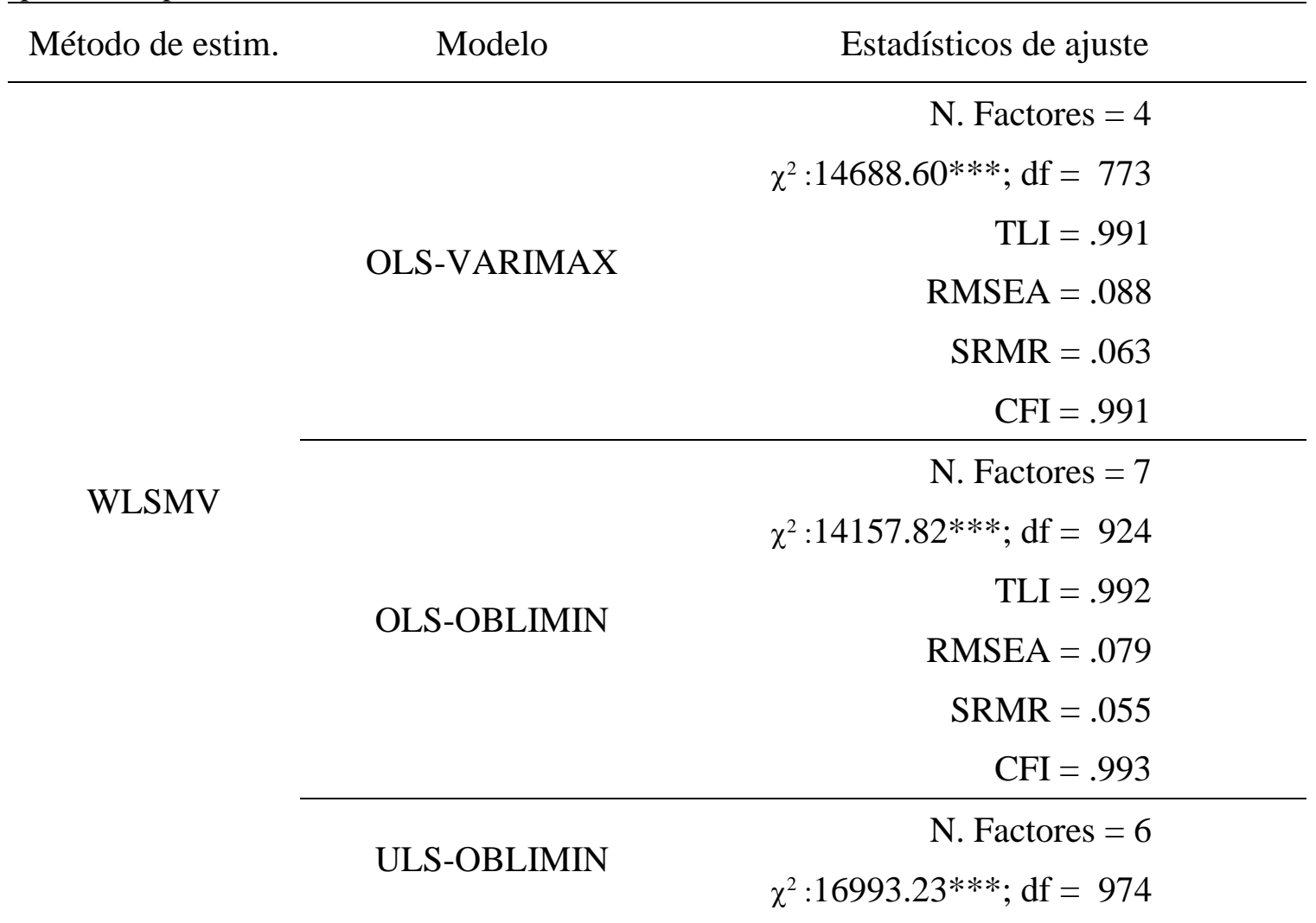

Factores condicionantes de la transferencia de la formación permanente del profesorado no universitario. Pamies-Berenguer M., Gomariz-Vicente M. A. y Cascales-Martínez A. 


TLI $=.991$
RMSEA $=.084$
SRMR $=.059$
CFI $=.992$
N. Factores $=7$
$\chi^{2}: 14522.81 * * ;$ df $=924$
TLI $=.992$
RMSEA $=.080$
SRMR $=.055$
CFI $=.993$
N. Factores $=8$
TMSEA $=.067$
SRMR $=.049$
CFI $=.995$

N. Factores $=7$

$\chi^{2}: 12358.47 * * * ; \mathrm{df}=798$

ML-OBLIMIN

$\mathrm{TLI}=.993$

RMSEA $=.079$

SRMR $=.057$

$\mathrm{CFI}=.993$

N. Factores $=6$

$\chi^{2}: 23715.68 * * * ; \mathrm{df}=1310$

MINRANK-PROMIN

$$
\mathrm{TLI}=.989
$$

RMSEA $=.086$

$\mathrm{SRMR}=.062$

$\mathrm{CFI}=.990$

N. Factores $=8$

$\chi^{2}: 70590.86^{* * *} ; \mathrm{df}=1456$

MODELO TEÓRICO

$\mathrm{TLI}=.970$

RMSEA $=.143$

$\mathrm{SRMR}=.103$

Factores condicionantes de la transferencia de la formación permanente del profesorado no universitario. Pamies-Berenguer M., Gomariz-Vicente M. A. y Cascales-Martínez A. 


CFI $=.971$
N. Factores $=1$
$\chi^{2}: 12358.47 * * ;$ df $=798$
TLI $=.993$
RMSEA $=.079$
SRMR $=.057$
CFI $=.993$

Nota. TLI $=$ Tucker-Lewis Index; RMSEA = Error cuadrático medio de aproximación; SRMR = Residuo estandarizado cuadrático medio; BIC = Bayesian Information Criterion.

Fuente: elaboración propia.

Los modelos, considerados adecuados, se sometieron a un estudio de la expresividad de las agrupaciones de ítems obtenidas y la consistencia interna de cada componente de la estructura. Entre las interpretaciones obtenidas por los diferentes modelos se optó por el modelo de 8 factores correspondiente a la combinación ULS-OBLIMIN. La Tabla 4 presenta la configuración de factores asociada al modelo seleccionado con expresión del nombre asignado a cada factor y la interpretación propuesta para cada uno de ellos. En dicha tabla, el número que aparece asociado a cada ítem se corresponde con el número de cuestión asignado en el cuestionario utilizado para la recogida de datos (PamiesBerenguer et al., 2020b).

Por otra parte, la Figura 2 recoge las estimaciones de los parámetros del modelo ajustado, donde se puede ver una comparativa entre las agrupaciones de ítems del modelo teórico adaptado y el seleccionado. Hay que tener presente que al utilizarse una estrategia de rotación oblicua se presentan relaciones de covarianza entre los diferentes factores.

Tabla 4.

Factores, agrupaciones de items e interpretación de los factores propuesto para el modelo obtenido mediante la combinación ULS-OBLIMIN de 8 factores.

\begin{tabular}{|c|c|c|l|c|c|}
\hline Fact. & $\mathrm{N}^{\mathrm{O}}$ Ítem & Identificador & \multicolumn{1}{|c|}{ Descriptor } & $\begin{array}{c}\text { Var. } \\
\text { Expl. }\end{array}$ & $\begin{array}{c}\text { Alfa } \\
\text { C. }\end{array}$ \\
\hline 1 & $\begin{array}{c}3 ; 1 ; 7 ; \\
2 ; 4 ; 5 ;\end{array}$ & $\begin{array}{c}\text { Diseño y } \\
\text { desarrollo de la la } \\
\text { formación }\end{array}$ & $\begin{array}{l}\text { Incluye la visión sobre los } \\
\text { aprendizajes realizados, el } \\
\text { impacto de los formadores, el } \\
\text { diseño de la actividad } \\
\text { formativa, la aportación de } \\
\text { los participantes y la aptitud } \\
\text { hacia la aplicación del } \\
\text { individuo. Se incluye como } \\
\text { producto de la formación } \\
\text { aquellos que intervienen } \\
\text { sobre la motivación del }\end{array}$ & $18.25 \%$ & .95 \\
\hline
\end{tabular}

Factores condicionantes de la transferencia de la formación permanente del profesorado no universitario. Pamies-Berenguer M., Gomariz-Vicente M. A. y Cascales-Martínez A. 


\begin{tabular}{|c|c|c|c|c|c|}
\hline & & & $\begin{array}{l}\text { participante para aplicar los } \\
\text { aprendizajes }\end{array}$ & & \\
\hline 2 & $\begin{array}{l}14 ; 10 ; \\
32 ; 34 ; \\
33 ; 35 ; \\
13 ; 36\end{array}$ & Autoeficacia & $\begin{array}{l}\text { Resume la autopercepción } \\
\text { hacia la capacidad, el deseo y } \\
\text { la determinación del } \\
\text { participante para aplicar los } \\
\text { aprendizajes de la formación } \\
\text { en su práctica docente. }\end{array}$ & $12.98 \%$ & .94 \\
\hline 3 & $\begin{array}{l}16 ; 17 \\
15 ; 18 \\
19 ; 20\end{array}$ & $\begin{array}{l}\text { Fomento de la } \\
\text { formación por } \\
\text { los órganos del } \\
\text { centro }\end{array}$ & $\begin{array}{l}\text { Recoge el impacto de la } \\
\text { visión sobre la actitud de los } \\
\text { responsables didácticos de las } \\
\text { materias impartidas o el nivel } \\
\text { en el que el participante } \\
\text { imparte docencia hacia la } \\
\text { aplicación de los aprendizajes } \\
\text { de la formación. Además se } \\
\text { recoge la visión de los } \\
\text { equipos docentes en los que } \\
\text { se encuentra inmerso el } \\
\text { participante. }\end{array}$ & $11.95 \%$ & .94 \\
\hline 4 & $\begin{array}{l}43 ; 41 ; \\
26 ; 53\end{array}$ & $\begin{array}{l}\text { Feedback de } \\
\text { los estudiantes } \\
\text { y familias }\end{array}$ & $\begin{array}{l}\text { Contempla la } \\
\text { retroalimentación, percibida } \\
\text { por el participante, que se } \\
\text { ofrece por parte del alumnado } \\
\text { (incluyendo sus familias) } \\
\text { cuando se incorporan los } \\
\text { aprendizajes derivados de la } \\
\text { formación en la docencia. }\end{array}$ & $8.40 \%$ & .85 \\
\hline 5 & $\begin{array}{l}38 ; 39 ; \\
25 ; 52 ; \\
24\end{array}$ & $\begin{array}{l}\text { Recursos del } \\
\text { entorno }\end{array}$ & $\begin{array}{l}\text { Mide la visión del } \\
\text { participante hacia las } \\
\text { facilidades que le ofrecen los } \\
\text { recursos (humanos, } \\
\text { materiales y económicos), } \\
\text { disponibles en el centro de } \\
\text { trabajo, a la hora de aplicar } \\
\text { los cambios propuestos en la } \\
\text { formación. }\end{array}$ & $7.69 \%$ & .88 \\
\hline 6 & $\begin{array}{l}22 ; 21 ; \\
23 ; 37\end{array}$ & $\begin{array}{l}\text { Resistencia al } \\
\text { cambio en el } \\
\text { centro de } \\
\text { trabajo }\end{array}$ & $\begin{array}{l}\text { Se mide el grado en el que el } \\
\text { participante percibe } \\
\text { resistencia para aplicar los } \\
\text { aprendizajes de la formación, } \\
\text { centrándose en la visión y } \\
\text { actitud a los cambios de los } \\
\text { compañeros docentes. }\end{array}$ & $7.38 \%$ & .89 \\
\hline 7 & $49 ; 48$ & $\begin{array}{l}\text { Seguimiento } \\
\text { de la } \\
\text { institución }\end{array}$ & $\begin{array}{l}\text { Recoge el esfuerzo que se } \\
\text { hace desde la entidad } \\
\text { organizadora de la formación }\end{array}$ & $5.78 \%$ & .93 \\
\hline
\end{tabular}

Factores condicionantes de la transferencia de la formación permanente del profesorado no universitario. Pamies-Berenguer M., Gomariz-Vicente M. A. y Cascales-Martínez A. 
RED. Revista de Educación a Distancia. Núm. 69, Vol. 22. Artíc. 4, 30-Ene-2022

DOI: http://dx.doi.org/10.6018/red.486801

\begin{tabular}{|c|c|c|l|c|c|}
\hline & formadora & $\begin{array}{l}\text { por realizar el seguimiento de } \\
\text { la aplicación de la formación. }\end{array}$ & & \\
\hline 8 & $46 ; 47$ & $\begin{array}{l}\text { Locus de } \\
\text { control externo }\end{array}$ & $\begin{array}{l}\text { Mide la resistencia, derivada } \\
\text { a elementos extornos, a la } \\
\text { aplicación de la formación a } \\
\text { su puesto de trabajo. }\end{array}$ & $3.99 \%$ & .83 \\
\hline \multicolumn{3}{|c|}{ Varianza explicada total: $76.41 \%$} & \\
\hline
\end{tabular}

Fuente: elaboración propia.

AGRUPACIÓN DE ÍTEMS EN EL MODELO FACTORIAL TEÓRICO
DIAGRAMA DE ESTRUCTURA DEL MODELO FACTORIAL PROPUESTO
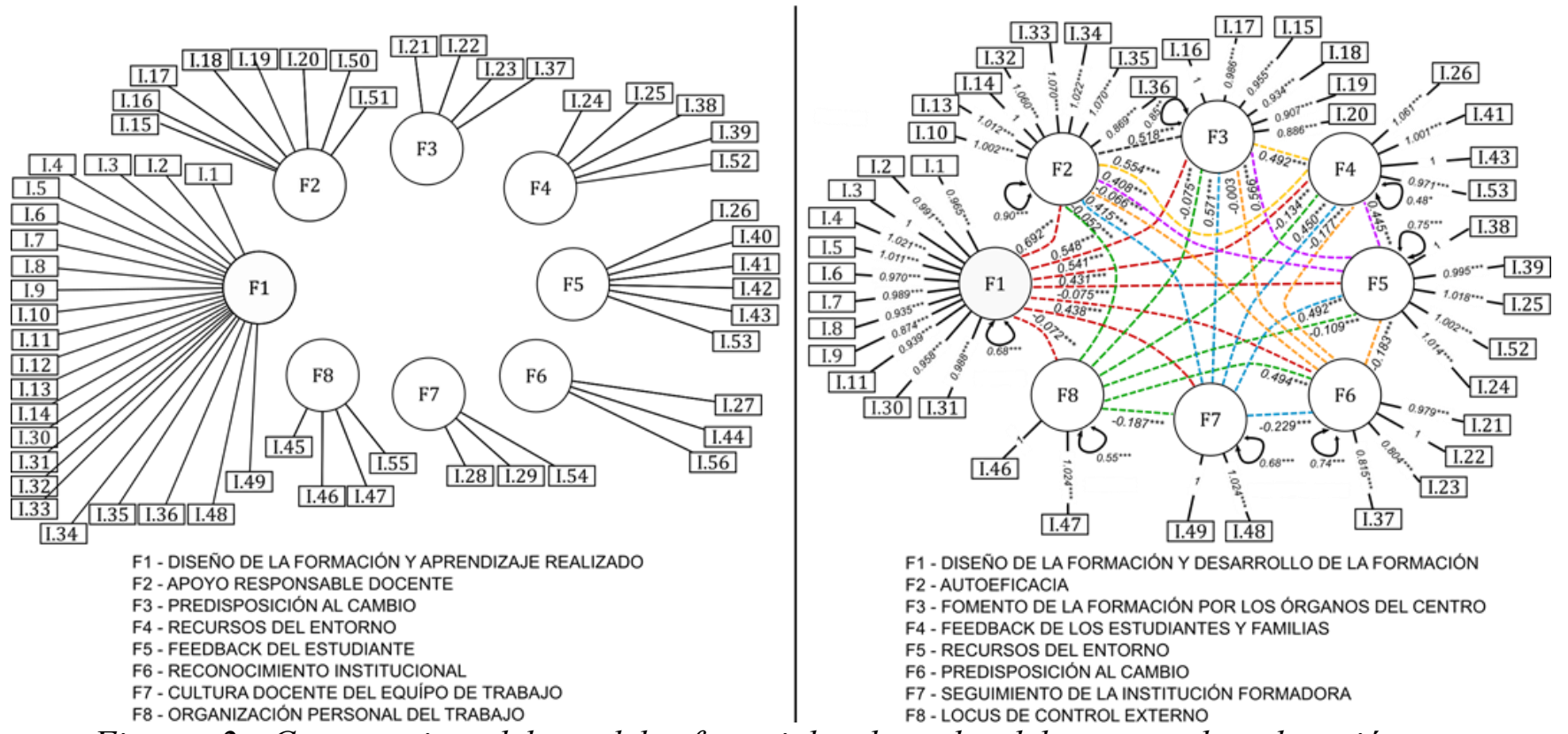

Figura 2. Comparativa del modelo factorial adaptado del marco de educación universitaria y el obtenido en la investigación.

La varianza explicada por el modelo propuesto ascendió al $76.41 \%$ y el análisis de la consistencia interna de cada agrupación de ítems, mediante el alfa de Cronbach (ver Tabla 4), ofreció unos resultados que los identifican como de Buenos o Excelentes dependiendo del caso.

\section{Discusión}

Tras el análisis de los resultados que se obtuvieron durante la fase de tratamiento de los datos, se ha dispuesto de la evidencia suficiente para poder abordar las respuestas a las preguntas de investigación planteadas inicialmente.

La primera cuestión (P1) planteaba la posibilidad de trasladar, de forma adaptada, el modelo factorial propuesto para el estudio de los factores de la transferencia de la formación permanente del docente universitario (Feixas et al., 2013a) al marco de la enseñanza no universitaria. En este sentido, el análisis realizado, haciendo uso de la adaptación del cuestionario y del modelo universitario al marco no universitario

Factores condicionantes de la transferencia de la formación permanente del profesorado no universitario. Pamies-Berenguer M., Gomariz-Vicente M. A. y Cascales-Martínez A. 
realizada en Pamies-Berenguer et al. (2020a, 2020b), nos ha permitido identificar un modelo de 8 factores que mantiene ciertas similitudes, en términos generales, con el primigenio (véase Figura 2).

El conjunto de factores identificados en el modelo propuesto (véase Tabla 4) se encuentra en consonancia con los identificados en otras investigaciones (Baldwin et al., 2017; Ester et al., 2019; Ford et al., 2018; Nazli y Khairudin, 2018; Ma et al., 2018; Tomàs-Folch y Duran-Bellonch, 2017; Yaqub y Dutta, 2021) en las que se destacan las asociaciones entre diferentes factores y la transferencia de la formación continua de los empleados de diferentes contextos profesionales. En particular, siguiendo la numeración fijada en la Tabla 4, el factor nro. 1 "Diseño de la formación" se ha posicionado como factor de influencia sobre la transferencia de la formación continua desde hace más de tres décadas (Baldwin y Ford, 1988) y se ha identificado desde una multitud de investigaciones realizadas en diferentes contextos tanto profesionales como formativos (Blume et al., 2019; Kiwanuka et al., 2020; Lacerenza et al., 2017; Ma et al., 2018; Sahoo y Mishra, 2019; Salleh et al., 2017). El factor nro. 2 "Autoeficacia", se ha descrito en la literatura sobre la transferencia como factor asociado a las características individuales de los participantes que inciden en la transferencia de la formación, se ha estudiado su impacto sobre la transferencia en función a la presencia de otras variables de mediación como la motivación, la satisfacción en el puesto de trabajo, etc. o su función como variable mediadora en relaciones de otras variables con la transferencia como la cultura de la organización, apoyo de la organización, etc. (Baldwin et al., 2017; Ford et al., 2018; Iqbal y Dastgeer, 2017; Na-Nan y Sanamthong, 2019; Rampun et al., 2020). Por otra parte, en Eid y Quinn (2017), Dirani (2017) o Quesada-Pallarès et al. (2018) se indica, asumiendo la variabilidad del contexto en el que se desarrollan las investigaciones, que los recursos que las organizaciones ponen a disposición de sus empleados condicionan la forma en la que éstos abordan el proceso de transferencia, lo que apoya la incorporación del factor nro. 5 "Recursos del entorno" en el modelo propuesto. La aparición del factor nro. 3 "Fomento de la formación por los órganos del centro" se interpreta como una componente contextualizada al marco de la educación del factor más general "Apoyo del supervisor" (Chauhan et al., 2017; Freitas et al., 2019; Park et al., 2018; Yaghi y Bates, 2020). El factor nro. 6 "Resistencia al cambio en el centro de trabajo" se asocia a una interpretación personal, realizada por los empleados, sobre las rutinas de trabajo en la organización en la que opera el empleado y, por lo tanto, puede verse como un componente del constructo más general de "Clima organizacional" señalado como un factor de influencia en la transferencia por varios autores (Banerjee et al., 2017; Imran et al., 2017; Northup, 2018; Rivai et al., 2019). El modelo dinámico propuesto por Blume et al. (2019) establece, como una de las fases del proceso de transferencia, la evaluación del desempeño y de las variables de salida que debe analizar el empleado tras producirse un intento de aplicación, indicando que dicha evaluación influye en los rasgos del individuo a la hora de enfrentarse a futuras situaciones de transferencia, lo que justifica la aparición en el modelo factorial propuesto de la dimensión nro. 4 "Feedback de los estudiantes y familias". Por otra parte, el factor nro. 7 "Seguimiento de la institución formadora" no es habitual encontrarlo como influyente en la transferencia en la literatura, ya que la fuente habitual de la organización que impulsa la formación es la propia organización en la que los empleados desarrollan sus funciones. Para el caso concreto del profesorado de enseñanzas no universitarias, la organización de las actividades estudiadas fue diseñada

Factores condicionantes de la transferencia de la formación permanente del profesorado no universitario. Pamies-Berenguer M., Gomariz-Vicente M. A. y Cascales-Martínez A. 
y desarrollada por la administración con competencias en educación, que podría ser percibida como un elemento externo al lugar donde se desarrollan las funciones propias del puesto de trabajo. Por último, se identifica (Factor nro. 8), la componente interna del constructo "Locus de control", que ha sido asociado con el proceso de transferencia en varios trabajos de investigación (Sahoo y Mishra, 2017; Tantanawat, 2020), que se centra en resaltar la visión del empleado sobre aquellos elementos de carácter externo que le son impuestos como limitantes para la aplicación de aprendizajes.

En términos comparativos, entre el modelo identificado en esta investigación y el teórico adaptado desde el marco universitario, se observa que algunos factores de éste se siguen identificando en mayor o menor grado (Diseño de la formación, Recursos del entorno). No obstante, la adaptación de los ítems del cuestionario y las agrupaciones de éstos en el modelo final han desvelado factores asociados al nuevo escenario (Autoeficacia, Locus de Control Externo, Seguimiento de la institución formadora...) o bien han matizado algunos de los que ya aparecían inicialmente (aparición de la familia, el fomento desde el centro educativo...). Así, podemos afirmar que la traslación del modelo adaptado del universitario al marco no universitario admite mejoras, quedando patente que los matices que diferencian ambas enseñanzas (contacto con el alumnado y familias, estructura de la formación continua o la presencia de cargos/órganos bien delimitados que ejercen cierta influencia durante el proceso de transferencia) juegan un papel principal tanto en la agrupación de las cuestiones como en la interpretación de los factores.

Por su parte, en la segunda cuestión de investigación (P2) se planteaba la posibilidad de encontrar un instrumento de medida para los factores que fuera compatible con el modelo adaptado o con el nuevo modelo. En la respuesta a la cuestión anterior se justificaba la mejora, en términos descriptivos, del nuevo modelo propuesto como resultado de nuestro análisis. Dicho modelo establece unas agrupaciones de ítems que difieren de las inicialmente obtenidas para el modelo universitario adaptado (ver Figura 2). Así, los ítems que han sobrevivido al proceso y sus agrupaciones en el nuevo modelo factorial conforman un nuevo instrumento de medida que se encuentra validado en contenido (Pamies-Berenguer et al., 2020a) y que, mediante este trabajo, queda validado en constructo.

\section{Conclusiones}

En términos generales, creemos que las particularidades del desarrollo profesional del profesorado de diferentes etapas y la idiosincrasia asociada al puesto de trabajo tienen su repercusión en la forma en la que se configuran los factores de influencia en la transferencia. Si bien, en este trabajo, se ha investigado la diferencia entre el nivel universitario y el no universitario, las diferencias podrían incluso producirse al introducir otros elementos diferenciadores como las políticas educativas desarrolladas en diferentes zonas geográficas. Estas diferencias ponen de manifiesto el valor de continuar con las investigaciones que permitan identificar factores de influencia en la transferencia en contextos de desarrollo profesional concretos que posibiliten el análisis comparativo entre situaciones afines que nos acerquen a un mayor entendimiento del problema planteado.

Factores condicionantes de la transferencia de la formación permanente del profesorado no universitario. Pamies-Berenguer M., Gomariz-Vicente M. A. y Cascales-Martínez A. 
Por otra parte, es importante destacar que la aportación de un marco factorial específico para el contexto de la enseñanza no universitaria abre la puerta a plantear nuevas preguntas de investigación para el mismo contexto. En esta línea, se plantea el estudio de modelos predictivos para la probabilidad de la transferencia en función a las puntuaciones factoriales. A este respecto, hay que tener en cuenta las limitaciones introducidas en los procesos inferenciales debidas a la utilización de un modelo que emerge desde un estudio diseñado con carácter transversal y que debería someterse a validaciones periódicas. No obstante, el modelo y los datos obtenidos tras la aplicación del cuestionario asociado junto con la aplicación de técnicas de aprendizaje supervisado pueden configurar una línea de investigación que proponga herramientas de predicción para la transferencia que presenten características adaptativas y que evolucionen a lo largo del tiempo incorporando ejemplos significativos a su base del conocimiento. De esta forma, se dotaría a los gestores de la formación, e incluso a los propios participantes, de un sistema de soporte en la toma de decisiones a la hora de actuar de forma consecuente sobre aquellos factores de influencia en la transferencia.

Por último, teniendo presente que el concepto que se ha manejado para la transferencia de la formación permanente exige el mantenimiento de los aprendizajes para su aplicación a lo largo del tiempo, los resultados obtenidos pueden favorecer el diseño de estudios de investigación sobre la dinámica de la transferencia, proporcionando un conjunto de ítems para medir la situación de cada factor en diferentes momentos del tiempo y plantear preguntas sobre su dinámica y el efecto de la misma sobre la transferencia.

\section{Agradecimientos}

Se agradece el apoyo y la colaboración recibida por la Consejería de Educación y Cultura de la Comunidad Autónoma de la Región de Murcia y por el Centro de Profesores y Recursos Región de Murcia.

Presentación del artículo: 17 de julio de 2021

Fecha de aprobación: 25 de septiembre de 2021

Fecha de publicación: 30 de enero de 2022

Pamies-Berenguer, M., Gomariz-Vicente, M. A. y Cascales-Martínez, A. (2022). Factores condicionantes de la transferencia de la formación permanente del profesorado no universitario. RED. Revista de educación a distancia, 22(69). http://dx.doi.org/10.6018/red.486801

Factores condicionantes de la transferencia de la formación permanente del profesorado no universitario. Pamies-Berenguer M., Gomariz-Vicente M. A. y Cascales-Martínez A. 


\section{Financiación}

Este trabajo no ha recibido ninguna subvención específica de los organismos de financiación en los sectores públicos, comerciales o sin fines de lucro.

\section{Referencias}

Aneas Novo, M. C. (2018). Valoración de la transferencia en las modalidades de autoformación y formación presencial. Innoeduca. International Journal of Technology and Educational Innovation, 4(2), 193-203. https://doi.org/10.24310/innoeduca.2018.v4i2.5303

Anwar, G. \& Abdullah, N. N. (2021). The impact of Human resource management practice on Organizational performance. International journal of Engineering, Business and Management (IJEBM), 5. https://doi.org/10.22161/ijebm.5.1.4

Baldwin, T. \& Ford, J. (1988). Transfer of training: A review and directions for future research. Personnel Psychology, 41(1), pp. 63-105. http://doi.org/10.1111/j.17446570.1988.tb00632.x

Baldwin, T.T., Ford, J. \& Blume, B. (2009). Transfer of training 1988-2008: and updated review and agenda for future research. International Review of Industrial and Organizational Psychology, Vol. 24, 41-70. https://doi.org/10.1002/9780470745267.ch2

Baldwin, T.T., Ford, J. \& Blume, B.D. (2017). The state of transfer of training research: moving toward more consumer-centric inquiry. Human Resource Development Quarterly, 28(1), 1-9. http://doi.org/10.1002/hrdq.212781

Banerjee, P., Gupta, R. \& Bates, R. (2017). Influence of organizational learning culture on knowledge worker's motivation to transfer training: testing moderating effects of learning transfer climate. Current Psychology, 36(3), 606-617. https://doi.org/10.1007/s12144-016-9449-8

Bawono, I. R. \& Purnomo, R. (2016). Antecedents and consequences of the transfer of training in public sector organizations. Journal of Economics, Business, and Accountancy Ventura, 19(2), 181-190. https://doi.org/10.14414/jebav.v19i2.568

Bernaards, C. A. \& Jennrich, R. I. (2005). Gradient Projection Algorithms and Software for Arbitrary Rotation Criteria in Factor Analysis. Educational and Psychological Measurement, 65, 676-696. https://doi.org/10.1177/0013164404272507

Blume, B.D., Ford, J. K., Baldwin, T. \& Huang, J. L. (2010). Transfer of Training: A Meta-Analytic Review. Journal of Management, 36(4), 1065-1105. https://doi.org/10.1177/0149206309352880

Blume, B. D., Ford, J. K., Surface, E. A. \& Olenick, J. (2019). A dynamic model of training transfer. Human Resource Management Review, 29(2), 270-283. https://doi.org/10.1016/j.hrmr.2017.11.004

Factores condicionantes de la transferencia de la formación permanente del profesorado no universitario. Pamies-Berenguer M., Gomariz-Vicente M. A. y Cascales-Martínez A. 
Buckworth, J. (2017). Issues in the Teaching Practicum. En G. Geng, P. Smith \& P. Black (Eds.), The Challenge of Teaching (pp. 9-17). (s.l): Springer Singapore. Recuperado de https://researchers.cdu.edu.au/en/publications/issues-in-the-teachingpracticum

Canto, J.L. (2016). Educación en las escuelas Normales: La formación docente en México - crítica, tendencia y propuesta. Revista Educação e Emancipação, 9(2), 6885. https://doi.org/10.18764/2358-4319.v9n2p68-85

Cejas-León, R. \& Navío-Gámez, A. (2020). Sobre la formación tecnopedagógica del profesorado. La visión de los expertos y formadores. Revista iberoamericana de educación superior, $\quad 11(31), \quad 150-164$. https://doi.org/10.22201/iisue.20072872e.2020.31.711

Chauhan, R., Ghosh, P., Rai, A. \& Kapoor, S. (2017). Improving transfer of training with transfer design: does supervisor support moderate the relationship? Journal of Workplace Learning. https://doi.org/10.1108/JWL-08-2016-0079

Comisión Europea/EACEA/Eurydice- (2018). La profesión docente en Europa: Acceso, progresión y apoyo. Informe de Eurydice. Luxemburgo: Oficina de Publicaciones de la Unión Europea. https://doi.org/10.2797/917260

Cubo, S., Marín, B. y Ramos, J. L. (2018). La investigación experimental. Métodos de investigación y análisis de datos en ciencias sociales y de la salud. Editorial Pirámide.

Díaz, G. A. T., Leyva, D. M. G., Yépez, C. A. P. \& Torres, P. J. P. (2021). Transferencia de conocimiento y los retos de la formación de ingenieros ante la globalización. Encuentros: Revista de Ciencias Humanas, Teoría Social y Pensamiento Crítico., (13). Recuperado de: https://dialnet.unirioja.es/descarga/articulo/7703903.pdf

Dirani, K. (2017). Understanding the process of transfer of training in a military context: Marching into new roles. Advances in Developing Human Resources, 19(1), 101-112. https://doi.org/10.1177/1523422316682929

Eid, A. \& Quinn, D. (2017). Factors predicting training transfer in health professionals participating in quality improvement educational interventions. BMC medical education, 17(1), 1-9. https://doi.org/10.1186/s12909-017-0866-7

Ester, H. R., Quesada-Pallarès, C. \& Pineda-Herrero, P. (2019). Diseño y pilotaje del METEnf: Modelo de Evaluación de los factores de Transferencia de la formación de los nuevos profesionales en Enfermería. Educación Médica, 22(5), 346-351. https://doi.org/10.1016/j.edumed.2019.09.005

Feixas, M., Duran, M.M., Fernández, I., Fernández, A., García San Pedro, M.J., Márquez, M.D., Pineda, P., Quesada, C., Sabaté, S., Tomàs, M., Zellweger, F. \& Lagos, P. (2013a). ¿Cómo medir la transferencia de la formación en Educación Superior?: el Cuestionario de Factores de Transferencia. Revista de Docencia Universitaria. REDU. Número monográfico dedicado a Formación docente del profesorado universitario, 11(3), Octubre-Diciembre, 219-248. https://doi.org/10.4995/redu.2013.5527.

Factores condicionantes de la transferencia de la formación permanente del profesorado no universitario. Pamies-Berenguer M., Gomariz-Vicente M. A. y Cascales-Martínez A. 
Feixas, M., Fernández, A., Lagos, P., Quesada, C. \& Sabaté, S. (2013b). Factores condicionantes de la transferencia de la formación docente en la universidad: un estudio sobre la transferencia de las competencias docentes. Infancia y aprendizaje, 36(3), 401-416. https://doi.org/10.1174/021037013807533034

Feixas, M., Lagos, P., Fernández, I. \& Sabaté, S. (2015). Modelos y tendencias en la investigación sobre efectividad, impacto y transferencia de la formación docente en educación superior. Educar, 51(1), 81-107. https://bit.ly/3rmqJIJ

Freitas, A. C., Silva, S. A. \& Santos, C. M. (2017). Predictors of safety training transfer support as in-role behavior of occupational health and safety professionals. European Journal of Training and Development, 41(9), 776-799. https://doi.org/10.1108/ejtd-03-2017-0024

Freitas, A. C., Silva, S. A. \& Santos, C. M. (2019). Safety training transfer: The roles of coworkers, supervisors, safety professionals, and felt responsibility. Journal of occupational health psychology, 24(1), 92. https://doi.org/10.1037/ocp0000125

Ford, J. K., Baldwin, T. T. \& Prasad, J. (2018). Transfer of training: The known and the unknown. Annual Review of Organizational Psychology and Organizational Behavior, 5, 201-225. https://doi.org/10.1146/annurev-orgpsych-032117-104443

Gafas González, C., Herrera Molina, A., Brossard Peña, E., Roque Herrera, Y., Ferrera, Y. \& Larramendi, R. (2018). El docente de tercer nivel en las ciencias de la salud. Contexto ecuatoriano. Educación Médica. 19(1), 34-8. https://doi.org/10.1016/j.edumed.2016.08.006

García, M. I. B., Castillo, V. D. V. L. \& Camacho, R. V. S. (2020). Formación docente universitaria, desde una mirada diversa y crítica de sus actores. Educación Superior, 30, 11-28. https://bit.ly/3uUROoB

García, O., De León, P., Mora, R.P. y Zea, A.M. (2018). Los profesores-investigadores universitarios y sus motivaciones para transferir conocimiento. Revista Electrónica de Investigación Educativa 20(3), 43-55. https://doi.org/10.24320/redie.2018.20.3.1754.

Govaerts, N., Kyndt, E., Vreye, S. \& Dochy, F. (2017). A Supervisors' Perspective on Their Role in Transfer of Training. Human Resource Development Quarterly, 28, 515-552. https://doi.org/10.1002/hrdq.21286

Grossman, R. \& Salas, E. (2011). The transfer of training what really matters. International Journal of Training and Development, 15(2), 103-120. https://doi.org/10.1111/j.1468-2419.2011.00373.x

Hicks, O. (1999). Integration of central and departmental development -reflections from Australian universities. International Journal for Academic Development, 4(1), 43-51. https://doi.org/10.1080/1360144990040107

Hughes, A. (2016). A meta-analytic integration of what matters in training transfer. Electronic Theses and Dissertations. Recuperado de https://bit.ly/3sTO0lp

Hu, L. T. \& Bentler, P. M. (1999). Cutoff criteria for fit indexes in covariance structure analysis: Conventional criteria versus new alternatives. Structural equation

Factores condicionantes de la transferencia de la formación permanente del profesorado no universitario. Pamies-Berenguer M., Gomariz-Vicente M. A. y Cascales-Martínez A. 


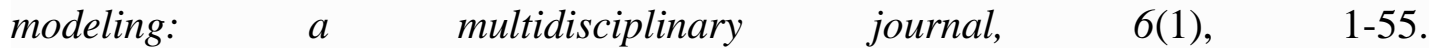
https://doi.org/10.1080/10705519909540118

Imran, K. \& Nazir, N. (2017). An Empirical Examination Of The Effects Of Personality Traits And Transfer Climate Factors On Transfer Of Training \& Motivation: A Longitudinal Study. Studies in Business and Economics, 12(3), 92-111. https://doi.org/10.1515/sbe-2017-0039

Iqbal, K. \& Dastgeer, G. (2017). Impact of self-efficacy and retention on transfer of training: The mediating role of motivation to transfer. Journal of Management Developmen, 36(10), 1270-1282. https://doi.org/10.1108/JMD-06-2015-0087

Izquierdo, I., Olea, J. \& Abad, F. J. (2014). El análisis factorial exploratorio en estudios de validación: Usos y recomendaciones. Psicothema, 26(3), 395-400. https://doi.org/10.22507/rli.v15n2a1

Kiwanuka, J., Miiro, R. F., Matsiko, F. B. \& Nkalubo, S. (2020). Using the Learning Transfer System Inventory to test the effects of trainee and training design characteristics on the transfer of agricultural training in Uganda. International Journal of Training and Development, 24(4), 374-383. https://doi.org/10.1111/ijtd.12202

Kleinbaum, D. G., Kupper, L. L. \& Muller, K. E. (1988). Applied regression analysis and other multivariable. PWS-Kent Publishing Company.

Lacerenza, C. N., Reyes, D. L., Marlow, S. L., Joseph, D. L. \& Salas, E. (2017). Leadership training design, delivery, and implementation: A meta-analysis. Journal of Applied Psychology, 102(12), 1686-1718. http://dx.doi.org/10.1037/ap10000241

Lüdecke D, Ben-Shachar M, Patil I. \& Makowski, D. (2020). Parameters: Extracting, Computing and Exploring the Parameters of Statistical Models using R. Journal of Open Source Software, 5(53), 2445. https://doi.org/10.21105/joss.02445

Ma, F., Bai, Y., Bai, Y., Ma, W., Yang, X. \& Li, J. (2018). Factors influencing training transfer in nursing profession: a qualitative study. BMC medical education, 18(1), 19. https://doi.org/10.1186/s12909-018-1149-7

Mardia, K. V. (1970). Measures of multivariate skewness and kurtosis with applications. Biometrika, 57(3), 519-530. https://doi.org/10.1093/biomet/57.3.519

Mason R.L., Gunst R.F. \& Hess, J.L. (1989). Statistical Design and Analysis of Experiments: Applications to Engineering and Science. Wiley.

Nafukho, F.M., Alfred, M., Chakraborty, M., Johnson, M. \& Cherrstrom, C.A. (2017). Predicting workplace transfer of learning. European Journal of Training and Development, 41(4), 327-353. http://doi.org/10.1108/EJTD-10-2016-0079

Na-Nan, K. \& Sanamthong, E. (2019). Self-efficacy and employee job performance: Mediating effects of perceived workplace support, motivation to transfer and transfer of training. International Journal of Quality \& Reliability Management, 37(1), 1-17. https://doi.org/10.1108/IJQRM-01-2019-0013

Navarro-González D. \& Lorenzo-Seva, U. (2020). EFA.MRFA: Dimensionality Assessment Using Minimum Rank Factor Analysis. R package version 1.0.9. https://CRAN.R-project.org/package=EFA.MRFA

Factores condicionantes de la transferencia de la formación permanente del profesorado no universitario. Pamies-Berenguer M., Gomariz-Vicente M. A. y Cascales-Martínez A. 
Nazli, N. N. N. N. \& Khairudin, S. M. H. S. (2018). The factors that influence transfer of training and its effect on organizational citizenship behaviour: Evidence from Malaysia civil defence force. Journal of Workplace Learning. https://doi.org/10.1108/JWL-09-2017-0080

Newstrom, J. W. (1986). Leveraging management development through the management of transfer. Journal of Management Development, 5(5), 33-45. https://doi.org/10.1108/eb051628

Northup, J. (2018). Strategies to Develop Skills for Positive Training Transfer. Adult Higher Education Alliance.

Orden 6771/2018, de 30 de octubre, de la Consejería de Educación, Juventud y Deportes, por la que se aprueba el Plan Trienal de Formación Permanente del Profesorado 2018-2021. (2018). Boletín Oficial de la Región de Murcia (BORM), 260, de 10 de noviembre de 2018, 28402-28436. https://www.borm.es/borm/documento?obj=anu\&id=771911

Pamies-Berenguer, M., Cascales-Martínez, A. \& Gomariz-Vicente, M. Á. (2020a). La validación de expertos en el proceso de adaptación de cuestionarios. El cuestionario de transferencia de la formación del profesorado no universitario. En R. Roig-Vila (Ed.), La docencia en la Enseñanza Superior. Nuevas aportaciones desde la investigación e innovación educativas (pp. 1410-1420). Octaedro.

Pamies-Berenguer, M., Cascales-Martínez, A. \& Gomariz-Vicente, M. Á. (2020b). Cuestionario de transferencia de la formación permanente del profesorado no universitario de la Comunidad Autónoma de la Región de Murcia. Editum. Recuperado en: http://hdl.handle.net/10201/93281

Park, S., Kang, H. S. T. \& Kim, E. J. (2018). The role of supervisor support on employees' training and job performance: an empirical study. European Journal of Training and Development. https://doi.org/10.1108/EJTD-06-2017-0054

Parra Robledo, R. \& Ruiz Bueno, C. (2020). Transferencia de la formación en competencias de liderazgo escolar en una universidad chilena. Revista Educación, 44(2), 138-153. http://dx.doi.org/10.15517/revedu.v44i2.40279

Poell, R. F. (2017). Time to 'flip' the training transfer tradition: Employees create learning paths strategically. Human Resource Development Quarterly, 28(1), 9-15. https://doi.org/10.1002/hrdq.21279

Poell, R. F. \& van der Krogt, F. J. (2003). Project-based learning in organizations: Towards a methodology for learning in groups. The Journal of Workplace Learning, 15(5), 217-228. https://doi.org/10.1108/13665620310483912

Quesada-Pallarès, C., Rebollar-Sánchez, E. M., Fernández-de-Álava, M. \& Ciraso, A. (2018). Is in-service teacher training useful? Applying the transfer of learning factors model in mexican schools. In L. Caudle (Ed.), Teachers and Teaching: Global Practices, Challenges and Prospects ( $1^{\text {st }}$ ed., pp. 1-24).

$\mathrm{R}$ Core Team (2020). R: A language and environment for statistical computing. $\mathrm{R}$ Foundation for Statistical Computing, Vienna, Austria. https://www.R-project.org/.

Factores condicionantes de la transferencia de la formación permanente del profesorado no universitario. Pamies-Berenguer M., Gomariz-Vicente M. A. y Cascales-Martínez A. 
Rampun, R., Zainol, Z. \& Tajuddin, D. (2020). The effects of training transfer on training program evaluation and effectiveness of training program. Management Research Journal, 9, 43-53. https://doi.org/10.37134/mrj.vol9.sp.4.2020

Revelle, W. (2020). Psych: Procedures for Personality and Psychological Research. Northwestern University, Evanston, Illinois, USA, https://CRAN.Rproject.org/package $=$ psych Version $=2.0 .7$

Rivai, R., Gani, M. U. \& Murfat, M. Z. (2019). Organizational culture and organizational climate as a determinant of motivation and teacher performance. Advances in Social Sciences Research Journal, 6(2). https://doi.org/10.14738/assrj.62.6267

Rosseel, Y. (2012). lavaan: An R Package for Structural Equation Modeling. Journal of Statistical Software, 48(2), 1-36. https://doi.org/10.18637/jss.v048.i02

Sahoo, M. \& Mishra, S. (2017). Training evaluation and motivation to transfer trainingA review of literature.Parikalpana: KIIT Journal of Management, 13(2), 17-28.

Sahoo, M. \& Mishra, S. (2019). Effects of trainee characteristics, training attitudes and training need analysis on motivation to transfer training. Management Research Review, 13(2), 17-28. https://doi.org/10.23862/kiit-parikalpana/2017/v13/i2/164517

Salas, E. \& Stagl, K. C. (2009). Design training systematically and follow the science of training. Handbook of principles of organizational behavior: Indispensible knowledge for evidence-based management.

Salazar-Gómez, E. \& Tobon, S. (2018). Análisis documental del proceso de formación docente acorde con la sociedad del conocimiento. Revista Espacios, 39(53).

Salleh, N. S. N. M., Amin, W. A. A. W. M. \& Mamat, I. (2017). Employee Readiness, Training Design and Work Environment in Influencing Training Transfer Among Academic Staffs of Uitm. International Journal of Academic Research in Business and Social Sciences, 7(10), 275-290. https://doi.org/10.6007/ijarbss/v7-i10/3376

Tantanawat, S. (2020). Individual Characteristic Predictors for Training Effectiveness in Thailand: A Study of Internal Locus of Control and Self-Efficacy. Journal of Community Development Research (Humanities and Social Sciences), 13(4), 1-8. https://doi.org/10.14456/jcdr-hs.2020.31

Tomàs-Folch, M. \& Duran-Bellonch, M. (2017). Comprendiendo los factores que afectan la transferencia de la formación permanente del profesorado. Propuestas de mejora. Revista Electrónica Interuniversitaria de Formación del Profesorado, 20(1), 145-157. https://doi.org/10.6018/reifop/20.1.24059

Vélez Álvarez, C., Jaramillo Ángel, C.P. y Giraldo Osorio, A. (2018). Docenciaservicio: responsabilidad social en la formación del talento humano en salud en Colombia. Educación Médica,19(52), 179-86.

Yaghi, A. \& Bates, R. (2020). The role of supervisor and peer support in training transfer in institutions of higher education. International Journal of Training and Development, 24(2), 89-104. https://doi.org/10.1111/ijtd.12173

Factores condicionantes de la transferencia de la formación permanente del profesorado no universitario. Pamies-Berenguer M., Gomariz-Vicente M. A. y Cascales-Martínez A. 
Yaqub, Y., Singh, A. K. \& Dutta, T. (2021). An empirical study of factors influencing training transfer in the management training intervention. Journal of Workplace Learning, 33(5), 361-374. https://doi.org/10.1108/jwl-02-2020-0034 
RED. Revista de Educación a Distancia. Núm. 69, Vol. 22. Artíc. 4, 30-Ene-2022

DOI: http://dx.doi.org/10.6018/red.486801

\section{Apéndice. Cuestionario de transferencia de la formación del profesorado no universitario (Pamies-Berenguer et al., 2020b)}

\begin{tabular}{|c|c|}
\hline Ítem & Enunciado \\
\hline 1 & $\begin{array}{l}\text { Los/las formadores/as de la actividad me han proporcionado nuevas ideas para poder } \\
\text { aplicar en mi aula los aprendizajes alcanzados }\end{array}$ \\
\hline 2 & $\begin{array}{l}\text { Los ejemplos presentados durante la formación me han resultado útiles para la } \\
\text { aplicación en el aula. }\end{array}$ \\
\hline 3 & La formación me ha permitido aprender nuevos enfoques docentes. \\
\hline 4 & $\begin{array}{l}\text { El diseño de la actividad formativa me ha ayudado a incorporar los aprendizajes de la } \\
\text { formación en mi docencia. }\end{array}$ \\
\hline 5 & La formación me ha dado seguridad para introducir cambios en mi aula \\
\hline 6 & $\begin{array}{l}\text { Me voy a esforzar por aplicar los aprendizajes derivados de la formación en mi práctica } \\
\text { docente. }\end{array}$ \\
\hline 7 & $\begin{array}{l}\text { Gracias a la formación he aprendido nuevas formas de trabajar con los estudiantes en } \\
\text { clase }\end{array}$ \\
\hline 8 & $\begin{array}{l}\text { El seguimiento de los/las formadores/as me ha permitido aplicar los aprendizajes en mi } \\
\text { aula. }\end{array}$ \\
\hline 9 & $\begin{array}{l}\text { El diseño de la actividad formativa ha permitido que los/las participantes aportáramos } \\
\text { nuevas experiencias aplicables en el aula. }\end{array}$ \\
\hline 10 & Me siento capaz de utilizar los aprendizajes de la formación en mi práctica docente. \\
\hline 11 & En la formación he aprendido a reflexionar sobre lo que hago en mi práctica docente. \\
\hline 12 & Durante la formación he tenido la oportunidad de ir practicando lo aprendido. \\
\hline
\end{tabular}

Factores condicionantes de la transferencia de la formación permanente del profesorado no universitario. Pamies-Berenguer M., Gomariz-Vicente M. A. y Cascales-Martínez A. 
RED. Revista de Educación a Distancia. Núm. 69, Vol. 22. Artíc. 4, 30-Ene-2022

DOI: http://dx.doi.org/10.6018/red.486801

\begin{tabular}{|c|c|}
\hline 13 & $\begin{array}{l}\text { Deseo aplicar lo aprendido en la formación para observar mejoras en el aprendizaje de } \\
\text { mis estudiantes. }\end{array}$ \\
\hline 14 & Me siento capaz de incorporar en mis clases, las ideas aprendidas en la formación. \\
\hline 15 & $\begin{array}{l}\text { El/la Jefe/a de Departamento o el/la Coordinador/a de Tramo o Ciclo en su caso } \\
\text { promueve que aplique cambios en mi docencia. }\end{array}$ \\
\hline 16 & $\begin{array}{l}\text { El/la Jefe/a de Departamento o el/la Coordinador/a de Tramo o Ciclo en su caso se } \\
\text { interesa sobre cómo puedo mejorar mi práctica docente a partir de la formación } \\
\text { recibida. }\end{array}$ \\
\hline 17 & $\begin{array}{l}\text { El/la Jefe/a de Departamento o el/la Coordinador/a de Tramo o Ciclo en su caso valora } \\
\text { los cambios que introduzco en mi tarea docente como resultado de la formación. }\end{array}$ \\
\hline 18 & $\begin{array}{l}\text { El/la Jefe/a de Departamento o el/la Coordinador/a de Tramo o Ciclo en su caso hace } \\
\text { un seguimiento de las mejoras que incorporo en la práctica docente. }\end{array}$ \\
\hline 19 & $\begin{array}{l}\text { Puedo contar con el apoyo de el/la Jefe/a de Departamento o el/la Coordinador/a de } \\
\text { Tramo o Ciclo en su caso para aplicar lo que he aprendido en la formación. }\end{array}$ \\
\hline 20 & $\begin{array}{l}\text { Nuestro equipo docente apoya al profesorado cuando introduce mejoras en su docencia } \\
\text { debido a la formación recibida. }\end{array}$ \\
\hline 21 & $\begin{array}{l}\text { Encuentro resistencias en el profesorado de mi departamento o de mi equipo de } \\
\text { tramo/ciclo para aplicar lo aprendido en la formación. }\end{array}$ \\
\hline 22 & $\begin{array}{l}\text { Encuentro resistencias en el profesorado de mi Claustro para aplicar lo aprendido en la } \\
\text { formación. }\end{array}$ \\
\hline 23 & $\begin{array}{l}\text { Son necesarias grandes dosis de entusiasmo en mi Claustro de profesores para } \\
\text { introducir un cambio }\end{array}$ \\
\hline 24 & $\begin{array}{l}\text { El apoyo técnico que ofrece mi centro (RMI, Orientación...) me facilita aplicar lo } \\
\text { aprendido en la formación. }\end{array}$ \\
\hline 25 & $\begin{array}{l}\text { Los recursos humanos disponibles en mi centro (profesorado auxiliar, de apoyo, etc.) } \\
\text { me facilitan el poder utilizar las habilidades adquiridas en la formación. }\end{array}$ \\
\hline 26 & $\begin{array}{l}\text { Cuando pruebo las cosas nuevas que he aprendido en la formación, los estudiantes } \\
\text { tienen una percepción más positiva de mí como docente. }\end{array}$ \\
\hline
\end{tabular}

Factores condicionantes de la transferencia de la formación permanente del profesorado no universitario. Pamies-Berenguer M., Gomariz-Vicente M. A. y Cascales-Martínez A. 
RED. Revista de Educación a Distancia. Núm. 69, Vol. 22. Artíc. 4, 30-Ene-2022

DOI: http://dx.doi.org/10.6018/red.486801

\begin{tabular}{|c|c|}
\hline 27 & $\begin{array}{l}\text { La administración educativa de mi Comunidad Autónoma reconoce el esfuerzo que } \\
\text { realizo para implementar la formación. }\end{array}$ \\
\hline 28 & $\begin{array}{l}\text { Comparto con mi Departamento o Equipo de Tramo/Ciclo experiencias y materiales } \\
\text { fruto de la formación recibida. }\end{array}$ \\
\hline 29 & $\begin{array}{l}\text { Cuando tengo un problema en la aplicación de lo aprendido, consulto con algún } \\
\text { compañero de mi claustro. }\end{array}$ \\
\hline 30 & Los formadores me han servido de modelo para mi docencia \\
\hline 31 & La formación me ha permitido actualizar los conocimientos. \\
\hline 32 & Creo que voy a poder aplicar adecuadamente lo aprendido en la formación \\
\hline 33 & $\begin{array}{l}\text { Puedo aplicar a mi docencia las estrategias de enseñanza y aprendizaje utilizadas en el } \\
\text { curso de formación. }\end{array}$ \\
\hline 34 & Voy a dedicar parte de mi tiempo de docencia a aplicar lo aprendido en la formación. \\
\hline 35 & Gracias a la formación he profundizado en conocimientos que ya tenía. \\
\hline 36 & Me gustaría que la formación realizada me ayudara a mejorar mi docencia. \\
\hline 37 & $\begin{array}{l}\text { La tradición docente en mi centro dificulta la aplicación de las innovaciones que } \\
\text { provienen de la formación. }\end{array}$ \\
\hline 38 & $\begin{array}{l}\text { Las posibilidades de la infraestructura del centro (tecnología, mobiliario, distribución } \\
\text { del aula, etc.) me facilitan la aplicación de lo aprendido en la formación. }\end{array}$ \\
\hline 39 & $\begin{array}{l}\text { Los recursos económicos disponibles en mi centro permiten implementar lo aprendido } \\
\text { en la formación. }\end{array}$ \\
\hline 40 & Mi alumnado no aprecia los cambios que aplico como resultado de la formación. \\
\hline
\end{tabular}

Factores condicionantes de la transferencia de la formación permanente del profesorado no universitario. Pamies-Berenguer M., Gomariz-Vicente M. A. y Cascales-Martínez A. 
RED. Revista de Educación a Distancia. Núm. 69, Vol. 22. Artíc. 4, 30-Ene-2022

DOI: http://dx.doi.org/10.6018/red.486801

\begin{tabular}{|c|c|}
\hline 41 & $\begin{array}{l}\text { Los comentarios del alumnado me animan a probar las nuevas estrategias docentes } \\
\text { aprendidas en la formación. }\end{array}$ \\
\hline 42 & $\begin{array}{l}\text { El alumnado es reacio a trabajar con los nuevos métodos que he aprendido en la } \\
\text { formación. }\end{array}$ \\
\hline 43 & $\begin{array}{l}\text { El alumnado valora positivamente que lleve a cabo métodos de enseñanza innovadores } \\
\text { como resultado de la formación. }\end{array}$ \\
\hline 44 & Espero que esta formación sea valorada para mi promoción académica. \\
\hline 45 & Con mi dedicación horario puedo implementar lo aprendido en la actividad formativa. \\
\hline 46 & Mi carga de trabajo me impide dedicar tiempo a implementar lo aprendido. \\
\hline 47 & La presión laboral me limita a aplicar lo aprendido en la formación. \\
\hline 48 & $\begin{array}{l}\text { El/la directora/a de la actividad formativa se interesa por conocer la aplicación de mis } \\
\text { aprendizajes en el aula. }\end{array}$ \\
\hline 49 & $\begin{array}{l}\text { El/la directora/a de la actividad realiza un seguimiento de la aplicación de los } \\
\text { aprendizajes de la formación al aula. }\end{array}$ \\
\hline 50 & Desde el Equipo Directivo se incentiva la aplicación de cambios en la docencia. \\
\hline 51 & El Equipo Directivo fomenta la aplicación de la formación del profesorado en el aula \\
\hline 52 & $\begin{array}{l}\text { Los recursos disponibles en el Centro de Profesores me facilitan la aplicación de los } \\
\text { aprendizajes de la formación al aula }\end{array}$ \\
\hline 53 & $\begin{array}{l}\text { Cuando pruebo lo aprendido en la formación en mis clases, las familias tienen una } \\
\text { percepción más positiva de mí como docente. }\end{array}$ \\
\hline 54 & $\begin{array}{l}\text { Los programas educativos que se desarrollan en mi centro estimulan la aplicación de } \\
\text { cambio en la docencia }\end{array}$ \\
\hline
\end{tabular}

Factores condicionantes de la transferencia de la formación permanente del profesorado no universitario. Pamies-Berenguer M., Gomariz-Vicente M. A. y Cascales-Martínez A.

Página 29 de 30 
RED. Revista de Educación a Distancia. Núm. 69, Vol. 22. Artíc. 4, 30-Ene-2022

DOI: http://dx.doi.org/10.6018/red.486801

\begin{tabular}{|c|l|}
\hline 55 & $\begin{array}{l}\text { Cuando realizo una formación siempre trato de encontrar tiempo para reflexionar sobre } \\
\text { cómo puedo aplicar los aprendizajes en mi aula }\end{array}$ \\
\hline 56 & $\begin{array}{l}\text { Cuando pruebo lo aprendido en la formación en mis clases, percibo que mis } \\
\text { compañeros/as de claustro tienen una visión más positiva de mí como docente. }\end{array}$ \\
\hline
\end{tabular}

Factores condicionantes de la transferencia de la formación permanente del profesorado no universitario. Pamies-Berenguer M., Gomariz-Vicente M. A. y Cascales-Martínez A. 